\title{
A Structure-Reactivity Relationship for Single Walled Carbon Nanotubes Reacting with 4-Hydroxybenzene Diazonium Salt
}

Nitish Nair, Woo-Jae Kim, Monica L. Usrey and Michael. S. Strano*

Department of Chemical and Biomolecular Engineering, University of Illinois at Urbana-Champaign, Urbana, Illinois 61801

\section{Supplementary Material}

The Supplement contains the detailed derivation of the rate model that is used to describe the surface coverage absorption data for representative single walled carbon nanotubes (SWNT). A parity plot of the relative rate constants for the light and dark reactions has also been included. The relative rate constants have been plotted with their 95\% confidence intervals. It is obvious from the graph that there is no statistical difference between them in the two cases. 


\section{Model Development}

We have developed a rate model for the reaction between the diazonium reagent and the nanotube sample. After fitting the model to steady state absorption data, we obtained rate constants relative to the $(11,5)$ nanotube, which had the maximum rate constant. In order to describe the trend in reactivities with band gap, we calculated rate constants relative to the $(11,5)$ tube by using Marcus theory and Gerischer-Marcus theory. The use of both formalisms is predicated on the assumption that the adsorption step, which is mediated by charge transfer, is rate limiting. ${ }^{12}$

Rate Equations. Consider the following adsorption-reaction scheme, ${ }^{12}$ for each $(n, m)$ nanotube:

$$
D+\theta_{(n, m)} \stackrel{k_{A}^{(n, m)}}{\longrightarrow} A \theta_{(n, m)} \stackrel{k_{R}^{(n, m)}}{\longrightarrow} P \theta_{(n, m)}
$$

Here, $D$ denotes the diazonium molecule, while $\theta_{(n, m)}, A \theta_{(n, m)}$ and $P \theta_{(n, m)}$ refer to the vacant sites on the nanotube, the sites occupied by the adsorption intermediate and the sites occupied by the reaction product, respectively. The adsorption rate constant is $k_{A}^{(n, m)}$, and the reaction rate constant is $k_{R}^{(n, m)}$.

An overall mass balance on the reactor gives its volume $\left(V_{R}\right)$ as a function of the initial volume $\left(V_{0}\right)$, volumetric flow rate of diazonium $\left(v_{0}\right)$ and time $(t)$, assuming that the density of the solution remains constant.

$V_{R}=V_{0}+v_{0} t$

A site balance carried out on the nanotube surface at any instant of time requires that the total number of sites $\left(N_{T_{(n, m)}}\right)$ on a particular nanotube be the sum of the number of vacant sites $\left(N_{\theta_{(n, m)}}\right)$ and those occupied by the adsorbed $\left(N_{A \theta_{(n, m)}}\right)$ and reacted species $\left(N_{P \theta_{(n, m)}}\right)$.

$$
N_{T_{(n, m)}}=N_{\theta_{(n, m)}}+N_{A \theta_{(n, m)}}+N_{P \theta_{(n, m)}}
$$


Once the reagent has entered the reactor, it reacts with the nanotubes to different extents, depending on whether they are metallic or semiconducting. The balance for the number of moles of diazonium $\left(N_{D}\right)$ yields

$$
\frac{d N_{D}}{d t}=F_{D_{0}}-\sum_{(n, m)} k_{A}^{(n, m)} \frac{N_{D} N_{\theta_{(n, m)}}}{V_{R}}
$$

where $F_{D_{0}}$ denotes the molar flow rate of diazonium into the reactor. The magnitude of the rate of depletion of diazonium, $r_{D}$, is given by the sum on the right hand side of the equation. On substituting the value for $N_{\theta_{(n, m)}}$ from the site balance in Equation 2, we obtain

$$
\frac{d N_{D}}{d t}=F_{D_{0}}-\frac{N_{D}}{V_{R}} \sum_{(n, m)} k_{A}^{(n, m)}\left[N_{T_{(n, m)}}-N_{A \theta_{(n, m)}}-N_{P \theta_{(n, m)}}\right]
$$

In order to obtain an expression for $N_{A \theta_{(n, m)}}$, we use the pseudo steady state approximation, wherein the adsorbed species is consumed as soon as it is generated.

$$
\frac{d N_{A \theta_{(n, m)}}}{d t}=\frac{k_{A}^{(n, m)}}{V_{R}} N_{D} N_{\theta_{(n, m)}}-k_{R}^{(n, m)} N_{A \theta_{(n, m)}}=0
$$

Once again, invoking the site balance, we obtain the following relation:

$$
N_{A \theta_{(n, m)}}=\frac{k_{A}^{(n, m)} N_{D}\left(N_{T_{(n, m)}}-N_{P \theta_{(n, m)}}\right)}{k_{R}^{(n, m)} V_{R}+k_{A}^{(n, m)} N_{D}}
$$

The rate of product formation is given by

$$
\frac{d N_{P \theta_{(n, m)}}}{d t}=k_{R}^{(n, m)} N_{A \theta_{(n, m)}}=k_{R}^{(n, m)} \frac{k_{A}^{(n, m)} N_{D}\left(N_{T_{(n, m)}}-N_{P \theta_{(n, m)}}\right)}{k_{R}^{(n, m)} V_{R}+k_{A}^{(n, m)} N_{D}}
$$

Defining the surface coverage on a $(n, m)$ nanotube as $\gamma_{(n, m)}=\frac{N_{P \theta_{(n, m)}}}{N_{T_{(n, m)}}}$, Equation 5a can be modified to read as

$$
\frac{d \gamma_{(n, m)}}{d t}=\frac{k_{R}^{(n, m)} k_{A}^{(n, m)} N_{D}\left(1-\gamma_{(n, m)}\right)}{k_{R}^{(n, m)} V_{R}+k_{A}^{(n, m)} N_{D}}
$$


Equation $3 b$ for diazonium can now be rewritten in terms of $\gamma_{(n, m)}$.

$\frac{d N_{D}}{d t}=F_{D_{0}}-\frac{N_{D}}{V_{R}} \sum_{(n, m)} k_{A}^{(n, m)} N_{T_{(n, m)}}\left(1-\gamma_{(n, m)}\right) \frac{k_{R}^{(n, m)} V_{R}}{k_{R}^{(n, m)} V_{R}+k_{A}^{(n, m)} N_{D}}$

At this point, we use the assumption that the adsorption step is rate-limiting (i.e., $\left.k_{R}^{(n, m)}>k_{A}^{(n, m)}\right)$ to simplify the mole balances.

$\frac{d N_{D}}{d t}=F_{D_{0}}-\frac{N_{D}}{V_{R}} \sum_{(n, m)} k_{A}^{(n, m)} N_{T_{(n, m)}}\left(1-\gamma_{(n, m)}\right)$

$\frac{d \gamma_{(n, m)}}{d t}=\frac{k_{A}^{(n, m)}}{V_{R}} N_{D}\left(1-\gamma_{(n, m)}\right)$

Equation $7 \mathrm{~b}$ is the general form for all nanotubes that participate in the reaction. In order to reduce the set of unknown parameters, the total number of sites on each nanotube $\left(N_{T_{(n, m)}}\right)$ is expressed as a fraction $\left(\alpha_{(n, m)}\right)$ of the total number of sites present in solution $\left(N_{T}\right)$.

$N_{T_{(n, m)}}=\alpha_{(n, m)} N_{T}$

$\alpha_{(n, m)}=\frac{A_{(n, m)}^{0}}{\sum_{(n, m)} A_{(n, m)}^{0}}$

The deconvolution of the absorption spectrum of an unreacted SWNT solution produces peak areas of the spectral profiles corresponding to each nanotube. ${ }^{11}$ The peak area of a nanotube in the unreacted decant solution $\left(A_{(n, m)}^{0}\right)$ is assumed to be proportional to the total number of available reaction sites on that nanotube, since increasing the surface coverage decreases the peak area.. When normalized to the total area, the ratio is assumed to give the fraction of sites for a particular nanotube. 


\section{Parity Plot}

The relative rate constants were the fit parameters in the rate model that was used to fit the surface coverage data. The values from the analysis of the light and dark absorption spectra have been plotted against each other, along with their $95 \%$ confidence intervals.

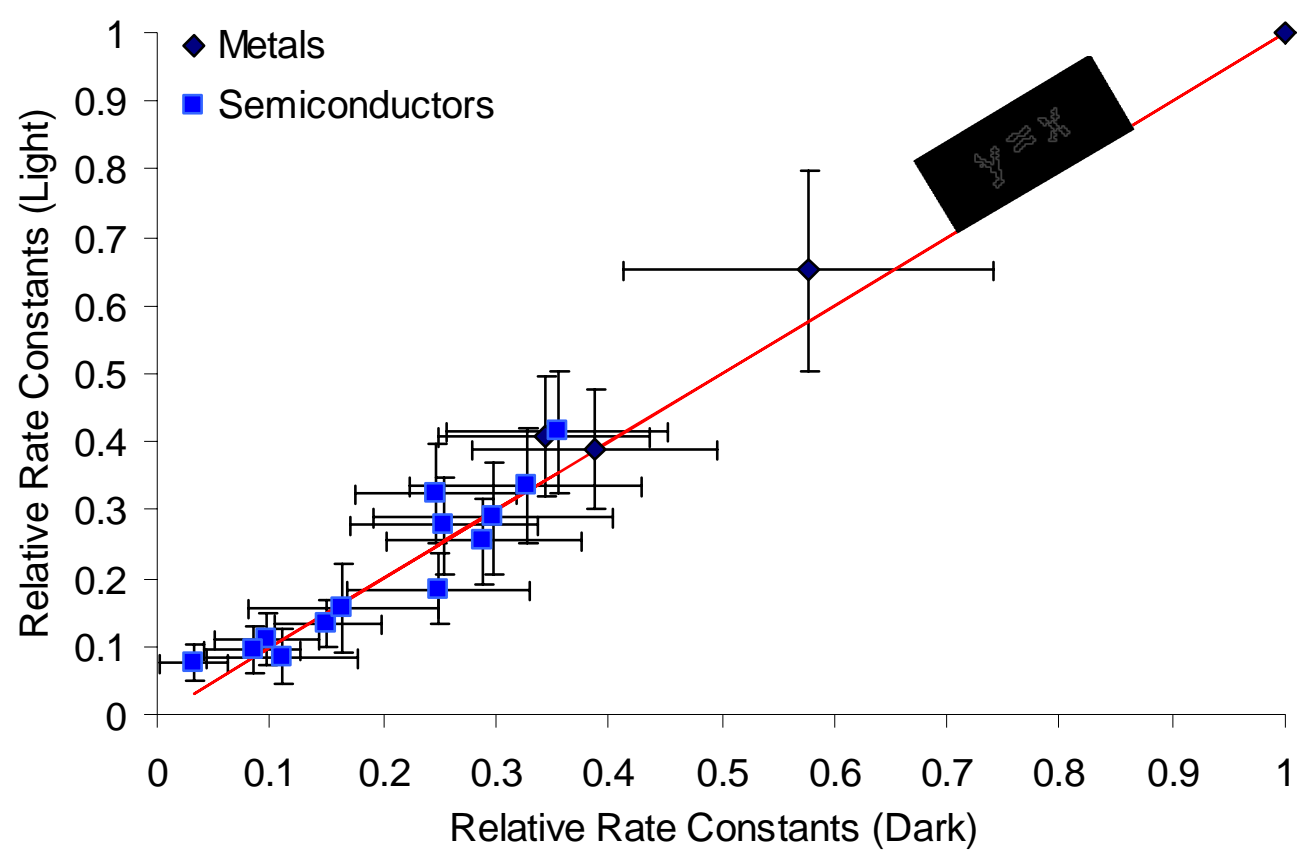

\section{Comparison of light and dark relative rate constants for semiconducting SWNT}

The plot below shows that the relative rate constants for the semiconductors are statistically similar for the light and dark reactions. There is a high degree of overlap between the $95 \%$ confidence intervals, especially for the large-diameter nanotubes.

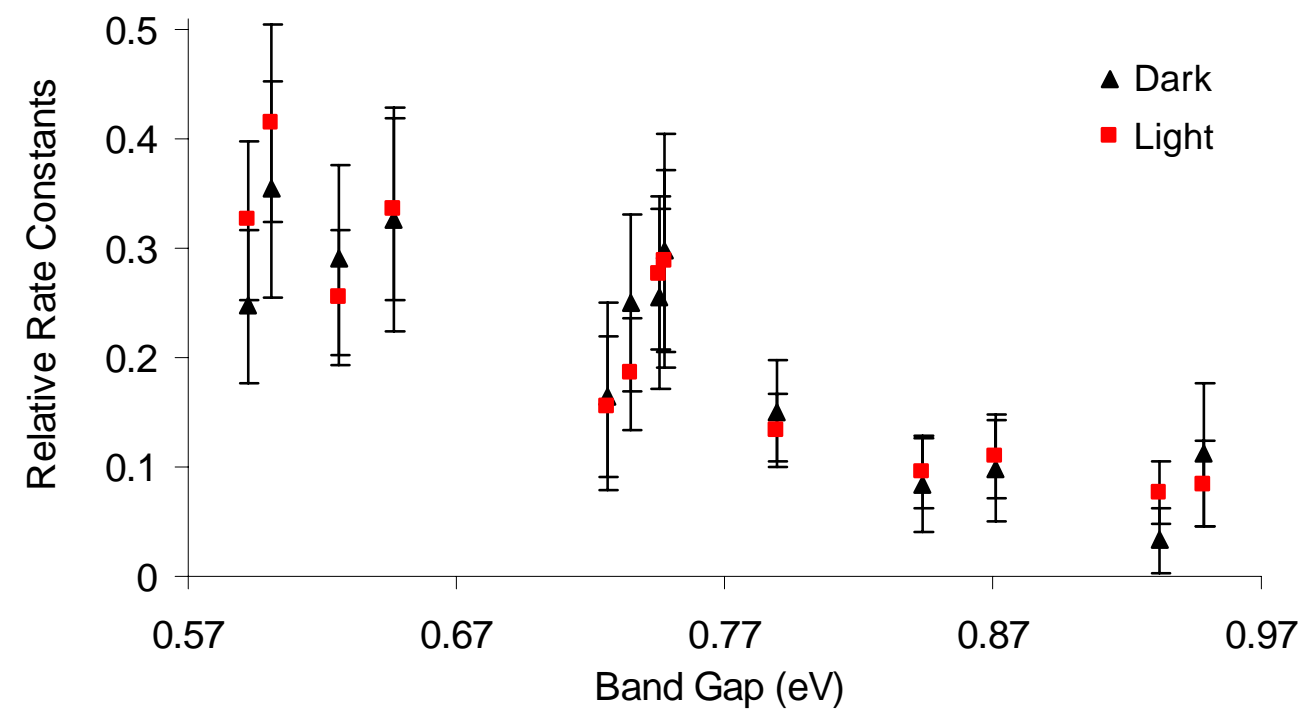

